\title{
Die Rolle der Kontrolle in Organisationen: Konzeptionen und Nutzenaspekte eines facettenreichen Begriffs
}

\author{
Peter Hensen ${ }^{1}$ \\ Online publiziert: 15 . April 2020 \\ (c) Der/die Autor(en) 2020
}

\section{Zusammenfassung}

Dieser Beitrag in der Zeitschrift Gruppe. Interaktion. Organisation (GIO) untersucht die funktionale Bedeutung von Kontrolle in Organisationen und arbeitet Nutzenaspekte für die Organisations- und Unternehmenspraxis heraus. Kontrolle als Begriff ist facettenreich. Er steht in enger Beziehung zu Macht und Handlung. Dazu gehören feststellende, reaktive und evaluative Tätigkeiten, die verallgemeinernd das Ziel verfolgen, ein Betriebs- und Organisationsgeschehen zu stabilisieren und zu optimieren. Konzipiert als technische Funktion dient Kontrolle der Erfüllung von Sachaufgaben; als Managementfunktion der Beherrschung von Unternehmensvorgängen. In einem konstruktivistischen Sinne erfüllt sie eine soziale Funktion des Gestaltens von Gemeinschaft. Kontrolle ist aber nicht nur sachbezogen zur Durchsetzung betrieblicher Interessen begründbar. Sie erfüllt darüber hinaus auch subjektive Nutzenfunktionen, die im wissenschaftlichen Diskurs und in der Organisationspraxis stärker betont werden sollten.

Schlüsselwörter Funktion $\cdot$ Führung $\cdot$ Kontrolle $\cdot$ Macht $\cdot$ Management $\cdot$ Organisation $\cdot$ Subjekt

\begin{abstract}
This paper in the journal Gruppe. Interaktion. Organisation (GIO) analyses the functional role of control in organisations and derives beneficial aspects for the organisational and corporate practice. Control is a multifaceted concept which is closely linked to power and action. The spectrum of „doing control“ usually includes measuring and determining as well as subsequent activities to evaluate and to react. The overarching concern is to stabilise and optimise the organisational performance. Control can be conceptualised as a technical function to fulfil operational tasks. In a second way it can be considered as a management function to achieve the corporate goals and put the plans into effect. From a constructivist perspective it can be conceived as a social function to create community. Control may be beneficial to pursue formal requirements, as well as to meet subjective needs. The latter aspect should be more emphasised in the scientific discourse and in organisational practice.
\end{abstract}

Keywords Control $\cdot$ Function $\cdot$ Leadership $\cdot$ Management $\cdot$ Organization $\cdot$ Power $\cdot$ Subject

\section{Einleitung}

Kontrolle ist ein Begriff, der alltags- und fachsprachlich viele Verwendungszusammenhänge kennt. Ohne jede Form von Kontrolle wäre ein Zusammenleben, wie wir es kennen, kaum denkbar und die Funktionsfähigkeit bzw. Zweckerfüllung von Leistungssystemen zumindest in Frage gestellt,

Prof. Dr. Peter Hensen, M.A., MBA

hensen@ash-berlin.eu

1 Alice Salomon Hochschule Berlin, Alice-Salomon-Platz 5, 12627 Berlin, Deutschland wobei über das Maß und den Wirkbereich im Einzelnen zu streiten ist. In Organisationen und organisierten Handlungssystemen ist sie „Teil des Programms“ und erscheint unentbehrlich im Umgang mit komplexen und kontingenten Arbeitswelten.

Der organisationale Kontrollbegriff hat eine natürliche Nähe zu herrschaftlich geprägter Macht (vgl. Richter 2014, S. 22). Kontrolle verspricht, dass Vorgaben erreicht und Regeln eingehalten werden. Wer kontrolliert, greift ein und kann Dinge nach bestimmten Vorstellungen beeinflussen. Sie wird deshalb nicht zwangsläufig von jedem Menschen als etwas Positives angesehen. Ganz im Gegenteil: „Nicht die Kontrolle zu haben“ oder „,von Kontrolle betroffen zu sein“, kann aber für den Einzelnen hochproblematisch sein, 
insbesondere wenn diese vom eigenen Umfeld entkoppelt, durch „fremde Dritte“ und sanktionsbewehrt einherkommt oder als sinnlos, zweckfremd und unverhältnismäßig wahrgenommen wird. Kontrolle steht für beides, Ordnung und Sicherheit, aber auch für Zwang und Einschränkung. Mit der Zughörigkeit zu einer organisierten Gemeinschaft wird die Ambivalenz dieses Begriffs für jeden unmittelbar erfahrbar. Die persönliche Wahrnehmung bestimmt ihre Deutung und den Umgang damit. Problembehaftetes Kontrollerleben und negative Begriffszuschreibungen sind Stressfaktoren, die zu kontraproduktivem Arbeitsverhalten führen können (Spector und Fox 2002; Meisler et al. 2017, S. 62; vgl. Hochwarter et al. 2020).

Die Unverrückbarkeit von Kontrolle und die Ambivalenz ihrer Deutung werfen die Frage auf, welche grundlegenden Anwendungs- und Bedeutungszusammenhänge dem Kontrollbegriff in Organisationen zugrunde liegen und welche Nutzenaspekte sich daraus destillieren lassen. Hierzu werden zunächst grundlegende Eigenschaften des Kontrollbegriffs skizziert. Es folgen historisch-narrative Betrachtungen, mit denen funktionale Konzeptionen isoliert und Bedeutungszusammenhänge des Kontrollbegriffs für die Organisations- und Unternehmenspraxis veranschaulicht werden. Aufbauend darauf werden sach- und subjektbezogene Nutzenaspekte identifiziert, die Organisationen und ihren Mitgliedern dabei helfen sollen, ein besseres Verständnis für die Kontrollabsichten und -erfordernisse im eigenen Arbeitsumfeld zu erlangen, eine neutrale Einordnung des Kontrollbegriffs in den organisationalen Gesamtzusammenhang vornehmen zu können und letztendlich eine positiv-konstruktive Grundhaltung zu diesem Begriff zu entwickeln. Abschließend wird eine kritische Einordnung vorgenommen und ein abschließendes Fazit gegeben.

\section{Eigenschaften des Kontrollbegriffs}

Augenscheinlich ist die Unbestimmtheit des Kontrollbegriffs. Diese ist nicht zuletzt darauf zurückführen, dass „Kontrolle“ ursprünglich dem angelsächsischen Sprachraum entnommen wurde und das Herkunftswort "Control“ nur unzureichend mit der alltagssprachlich oft verkürzt als Überwachung oder Inspektion verstandenen Kontrolltätigkeit beschrieben wird. Als Fachbegriff verteilt sich Kontrolle hierzulande auf die unterschiedlichsten Disziplinen und Anwendungszusammenhänge (vgl. Pries et al. 1990). Dazu gehören u. a. Bildungs- und Lerntheorien, betriebswirtschaftliche Konzepte der Unternehmensführung, Mechanismen der Mess-, Steuerungs- und Regelungstechnik, Fragen gesellschaftlicher und organisationsinterner Machtverteilung, insbesondere in Gestalt des professionsund organisationssoziologischen Autonomie-Begriffs, und nicht zuletzt die Absichten der Qualitätssicherung und des
Qualitätsmanagements (vgl. Ittel et al. 2014; Horváth et al. 2015; Krist 1997; Jürgens 1984; Freidson 1984; Juran 1995).

Im Organisationskontext steht Kontrolle begrifflich in wechselseitiger Beziehung und unauflösbarer Abhängigkeit zu Macht und Handlung. Beispielsweise ist jede erfolgreiche Handlung in gewisser Weise auf das Vorhandensein von Macht angewiesen, insofern mit dieser Handlung ein Wille durchgesetzt oder eine vorgängige Absicht realisiert wird. Auf einen solchen Machtbegriff, der im Kant'schen Sinne auf ein „Vermögen“ referenziert, ,welches großen Hindernissen überlegen ist" (Kant o.J.), ist besonders betriebliches Handeln in der Ungewissheit und unter Unsicherheit angewiesen. Ein solches Handeln, sofern es im Sinne der Zweckbestimmung erfolgreich sein soll, wird im organisationalen Kontext auch als Kontrolle bezeichnet (ausführlich dazu Matys 2014, S. 36 ff.). Kontrolle wird üblicherweise direkt und unmittelbar ausgeübt, z.B. durch Autorität und Sanktionierung (Prozessualität von Kontrolle). Sie kann aber auch indirekt und mittelbar wirken, ohne dass als solche definierte Kontrolltätigkeiten ausgeübt werden, z.B. durch Zustimmung und Gebundenheit. Insbesondere die Kategorie der Mittelbarkeit korrespondiert mit einem Machtbegriff, der im Luhmann'schen Sinne den Charakter eines symbolisch generalisierten Kommunikationsmediums trägt und der unter anderem dadurch geprägt ist, dass er durch seinen Symbolgehalt nachgeordnete Kontrolltätigkeiten quasi überflüssig erscheinen lässt (vgl. Ameln und Kramer 2012; Fischer 2004, S. 53 ff.). Eine solche mittelbare Kontrollwirkung baut rückkoppelnd auf erfahrbare Handlungserfolge und der Glaubwürdigkeit eines o. g. ,Vermögens" auf (Potenzialität von Kontrolle).

Als organisationaler Handlungsbegriff ist Kontrolle vom Macht- und Handlungsbegriff kaum zu isolieren. Verallgemeinernd und vereinfachend soll sie daher als machtgebundenes Organisationshandeln verstanden werden, durch das Festlegungen getroffen, Feststellungen vorgenommen und Dinge innerhalb definierter Wirk- und Handlungsbereiche (Kontrollbereiche) absichtsvoll beeinflusst werden. Der Kontrollbegriff verfügt sowohl über inhaltstheoretische als auch prozesstheoretische Anteile. $\mathrm{Zu}$ seinen wesentlichen Merkmalszügen gehören sicherlich:

- Absichten und Annahmen darüber, was innerhalb der Kontrollbereiche getan und erreicht werden soll bzw. welchen Zweck diese erfüllen (Soll-Vorstellungen);

- Mittel und Strukturen der direkten und indirekten Kontrollausübung (Kontrollsysteme und -instrumente); sowie

- situative oder geplante Aktivitäten (Kontrolltätigkeiten und -handlungen) zur Feststellung und Beeinflussung der Verwirklichung von Soll-Vorstellungen.

Kontrollsysteme und -instrumente bestehen üblicherweise aus Normen, Regeln, Vorschriften und Anweisungen 

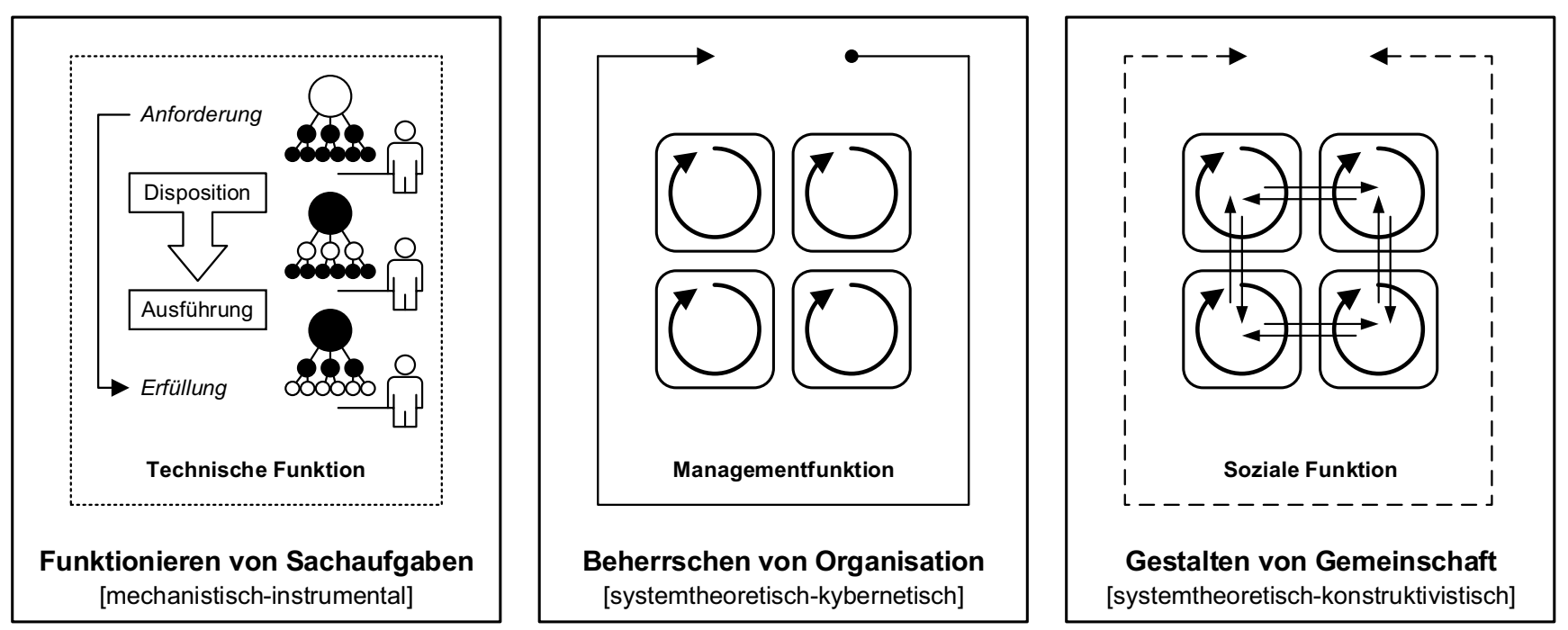

Abb. 1 Funktionale Konzeptionen von Kontrolle in Organisationen

oder aus Anreiz- und Zielsystemen. Mit derartigen Festlegungen werden die Soll-Vorstellungen operationalisiert. Zur Kontrollausübung gehören notwendigerweise Mess-, Überwachungs- und Prüfsysteme, mit denen im Zusammenspiel auf die Umsetzung und Einhaltung der getroffenen Festlegungen hingewirkt wird.

Kontrolltätigkeiten unterstützen die Verwirklichung festgelegter Soll-Vorstellungen. In einem engen Verständnis gehören hierzu Tätigkeiten der Ermittlung und Bestimmung von (Ist-)Zuständen, um einen Vergleich mit einzuhaltenden (Soll-)Festlegungen herbeizuführen (feststellende Kontrolltätigkeiten). Zu diesen Tätigkeiten gehört beispielsweise die Ermittlung von Prozesskennzahlen oder die Bestimmung von Verhaltensweisen in Form von dokumentierten Handlungen. Im Gegensatz zu einer rein ausführenden Messund Überwachungstätigkeit sind Kontrolltätigkeiten stets auf das Vorhandensein von operationalisierten Soll-Vorstellungen angewiesen (z. B. Unternehmensziele, Auditkriterien, Prozessvorgaben, Einhaltungsraten, Toleranzgrenzen).

In einem erweiterten Verständnis wird nicht nur die Übereinstimmung oder Abweichung von einer (Soll-)Festlegung bestimmt. Der weiter gefasste Kontrollbegriff zielt auf die Stabilisierung und Optimierung von Prozessen und Systemen, d.h. auf die absichtsvolle Beeinflussung des Kontrollbereichs. Zur Stabilisierung werden reaktive Maßnahmen ergriffen, die darauf gerichtet sind, die vorgesehenen Festlegungen einzuhalten bzw. festgestellten Abweichungen entgegenzuwirken (reaktive Kontrolltätigkeiten). Dabei kann es sich beispielsweise um Korrekturen zur Behebung von Fehlern oder um Korrekturmaßnahmen zur künftigen Vermeidung von aufgetretenen Fehlern handeln. Ausgangspunkt reaktiver Kontrolltätigkeiten sind erkennbare und beobachtete Problemlagen, beispielsweise in Gestalt einer festgestellten Normabweichung. Zur Op- timierung wird dagegen eine Bewertung bzw. Beurteilung eines Kontrollgegenstands hinsichtlich der vorgesehenen Funktions- und Wirkungsweise vorgenommen (evaluative Kontrolltätigkeiten). Im Gegensatz zu reaktiven haben evaluative Kontrolltätigkeiten proaktiven Charakter, d.h. sie kommen nicht ereignisorientiert oder fehlerbezogenen zur Anwendung, sondern werden systematisch und vorausschauend zur Identifikation von Verbesserungspotenzialen oder zur Gefahrenabwehr eingesetzt. Die Optimierung wird in der Regel durch Vorbeugungs- und Verbesserungsmaßnahmen erreicht. Es kann sich dabei um operative Folgeaktivitäten handeln (z. B. Modifikation von Prozessstandards) oder um strategische Maßnahmen mit organisations- und kulturbezogenen Veränderungen.

\section{Funktionale Konzeptionen}

Die hier skizzierten Eigenschaften sind das Ergebnis einer formalen Betrachtung des Kontrollbegriffs. Die formgebende Beschreibung taugt als Analyserahmen für die Bestimmung von Kontrolle im jeweiligen Anwendungsfeld. Darüber hinaus erscheinen Betrachtungen sinnvoll, die nicht nur eine theoretisch-methodische Beschreibung des Kontrollbegriffs liefern, sondern die funktionale Bedeutung von Kontrolle in Organisationen veranschaulichen bzw. einen Anwendungsbezug zur Arbeitswelt herstellen. Dazu werden im Folgenden drei Zugänge eröffnet.

\subsection{Kontrolle als technische Funktion}

Kontrolle als absichtsvolles Handeln ist wahrscheinlich so alt wie die Menschheit. Aufmerksamkeit und Relevanz in einem wissenschaftlichen Sinn erhielt der Begriff in beson- 
derer Weise Anfang des 20. Jahrhunderts mit der „Zweiten Industriellen Revolution“. Nach tayloristischem Vorbild organisierten sich Unternehmen und Produktionsbetriebe zunehmend arbeitsteilig, funktionsorientiert und hierarchisch. Gleichzeitig entwickelte sich eine Art Überwachungsbürokratie, die damit beschäftigt war, die Tätigkeit der Arbeiter und die Beschaffenheit von Waren bzw. Fertigungsteilen in der Produktion systematisch zu prüfen (vgl. Ketting 1999). Die Prüfung beschränkte sich zunächst auf Endkontrollen im Sinne einer Ergebnisprüfung, um fehlerhafte Produkte auszusortieren. Sie wurde später auf Zwischenprodukte ausgeweitet, um unerwünschte Abweichungen frühzeitig zu erkennen. In einem dritten Schritt kam die Beschaffenheitsprüfung der Zulieferprodukte hinzu, damit keine fehlerhaften Waren den Produktionsprozess gefährden (vgl. Schildknecht 1992; Schmitt und Pfeiffer 2015). Diese frühe Form systematisch-betrieblicher Kontrollausübung war gekennzeichnet durch die Trennung von disponierender und ausführender Arbeit. Verantwortung trägt alleinig die Unternehmensleitung, die mit hohem Aufwand Kontrolle ausübt. Die Produktion war beschäftigt mit Prüfen, Zählen, Sortieren und Reparieren, die Führungskräfte mit Überwachung und Disziplinierung der Beschäftigten (vgl. Matys 2014, S. $26 \mathrm{ff}$.). Ein Kontrollbegriff, der derart unmittelbar und direkt auf die Umsetzung angewiesener Arbeitsvollzüge und die Einhaltung vorgegebener Produktionsstandards gerichtet ist, entspricht einem mechanistisch-instrumentalen Verständnis von Arbeitsorganisation („Command-and-Control"-Logik). Kontrolle wird darin als technische Funktion zur Erfüllung von Sachaufgaben konzipiert (siehe Abb. 1).

Der Kontrollbegriff und mit ihm das Verständnis von Organisation und Unternehmensführung erfuhr Mitte des 20. Jahrhunderts einen Entwicklungsschub. Angefeuert durch die japanische und US-amerikanische Qualitätsbewegung traten Konzeptionen hervor, die als „Total Quality Control“ (Feigenbaum 1962) und "Company Wide Quality Control“" (Ishikawa 1985) bezeichnet wurden. Der Blick hob sich zunächst von der Produktion auf die Herstellungs- und Entwicklungsprozesse und später auch auf alle sonstigen Gelingensbedingungen. Damit wuchs ein durchgängigeres Verständnis von Kontrolle heran, das vermehrt vorausschauende und steuernde Elemente integrierte. Kontrolle musste nicht mehr nur organisiert werden, sie wurde zu einem Bestandteil organisationalen Handelns. Gleichzeitig wurde der Kontrollbegriff zunehmend unbestimmter. Die technische Funktion der Prüfung von Beschaffenheit bzw. der Überwachung des Arbeitsvollzugs wurde dadurch aber nicht bedeutungslos. Sie ist bloß um systemund handlungstheoretische Betrachtungen erweitert worden und lebt gewissermaßen in komplexeren Management- und Steuerungsideen weiter.

\subsection{Kontrolle als Managementfunktion}

Eine Neuorientierung erfährt der Kontrollbegriff ab Mitte des 20. Jahrhunderts durch die Integration systemtheoretischer Denkansätze in die Managementlehre (vgl. Ulrich 1968; Bleicher 1972). Im Zentrum dieses „Systemdenkens" steht die Erkenntnis, dass Unternehmen und Organisationen komplexe soziale Systeme sind, die in Austausch- und Wechselbeziehungen mit sich und ihrer Umwelt stehen. Komplexität und Unsicherheit wird begegnet mit Ordnungsmustern und Regelkreisen, mit denen das Zusammenspiel der Systemkomponenten aufgabenbezogen abgestimmt bzw. sinn- und zweckgebunden „beherrschbar“ werden soll. Die ordnungsgebenden Aufgaben drücken sich als Managementfunktionen aus.

Planung übt in solchen Ordnungsmustern üblicherweise die Primärfunktion aus, der sich die anderen Managementfunktionen unterordnen (Steinmann et al. 2013). Zu einer systematischen Planung gehört das sorgfältige Nachdenken, das Festlegen von Inhalten und Zielen (z.B. Unternehmens- und Qualitätszielen) sowie die Beantwortung der praxisrelevanten Frage, wie die Inhalte und Ziele umgesetzt werden. Um Prozesse, und damit auch die Leistungen und Ergebnisse der Organisation, vorausschauend und durch das Vorwegdenken von Schwierigkeiten realisieren zu können, sollte eine solche Planung notwendigerweise Kontrolltätigkeiten vorsehen, mit denen festgestellt wird, ob und inwieweit die getroffenen Festlegungen im Einzelnen erfüllt werden, aber auch, wie zur Stabilisierung und ggf. Optimierung des Kontrollbereichs beigetragen werden kann. Auf diese Weise ergänzen sich Planung und Kontrolle und verbinden sich gewissermaßen zu einer Art „ZwillingsFunktion“. Ihre wechselseitige Abhängigkeit wird durch die Feststellung von Wild (1982, S. 44), dass „Planung ohne Kontrolle sinnlos und Kontrolle ohne Planung unmöglich“ sei, treffend zum Ausdruck gebracht. Zwischen Planung und Kontrolle tritt naturgemäß die zweckgerichtete Ausführung, d.h. die sachbezogene (z.B. Aufbau eines Handlungsgerüsts, Koordination von Prozessen) und personenbezogene (z. B. Personaleinsatz, interaktionelle Mitarbeiterführung) Verwirklichung der festgelegten Inhalte und Ziele. Diese Managementfunktionen bilden eine dynamische und aufeinander aufbauende Abfolge von Aufgaben, die durch Entscheidungs- und Koordinationsaufgaben mit- und untereinander verbunden sind (vgl. Koontz und O’Donnell 1955; Steinmann et al. 2013, S. 12).

Innerhalb solcher auch als „kybernetisch“ bezeichneten Ordnungsmuster (vgl. Mirow 1969; Liesegang und Ullmann 1994) ist Kontrolle die „Stunde der Wahrheit“, die sodann zu einer „Stunde des Handelns“ wird. Der Stellenwert eines erweiterten, d.h. stabilisierenden und optimierenden Kontrollbegriffs lässt sich am kybernetischen $R e$ gelkreismodell des Qualitätsmanagements, dem sogenann- 
Abb. 2 Kontrolle als Managementfunktion am Beispiel des PDSA-Zyklus

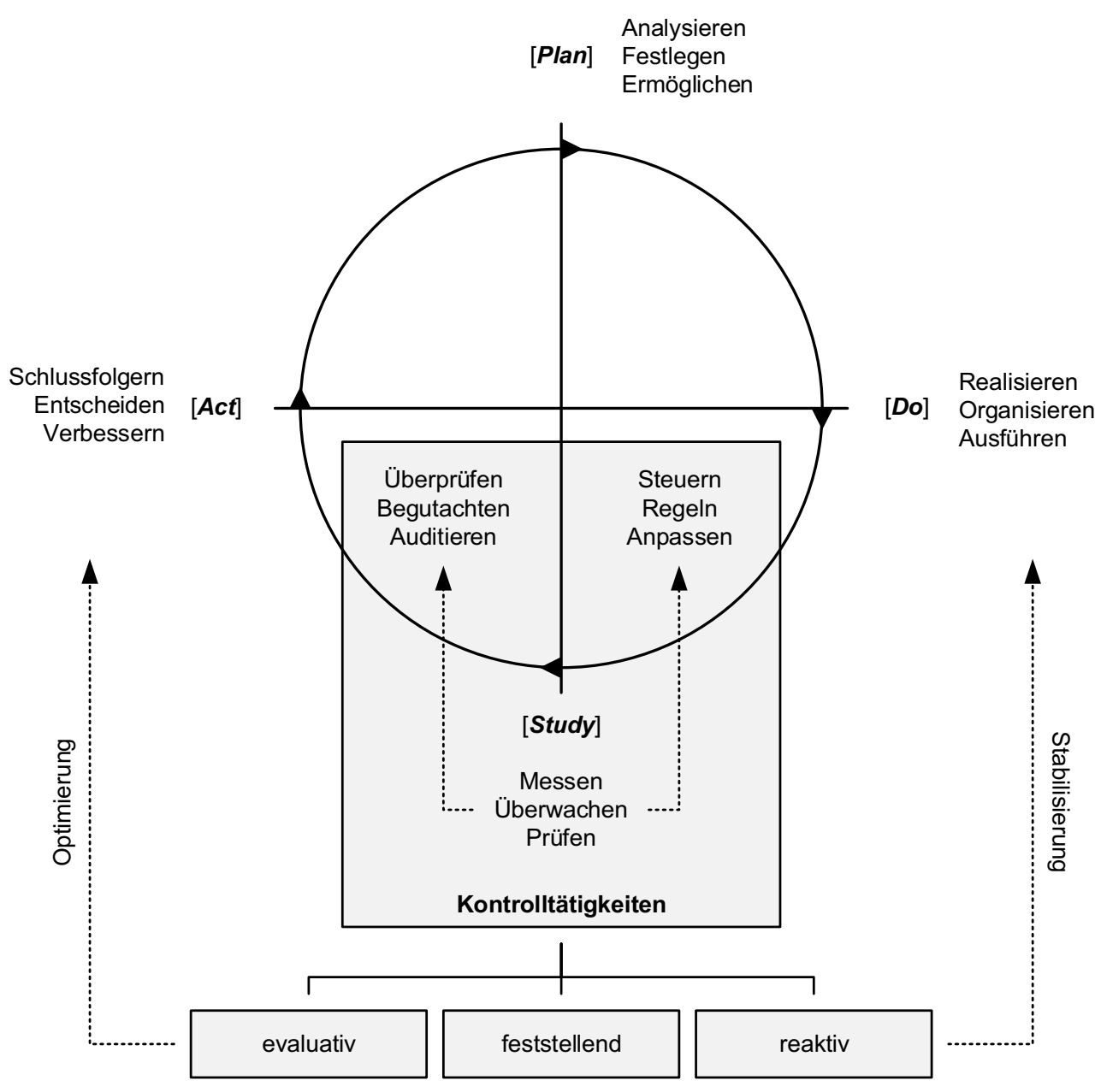

ten Plan-Do-Study-Act-Kreislauf (PDSA-Zyklus), illustrieren. In diesem auch als „Learning-and-Improvement-Cycle" (vgl. Deming 1994; Shewhart 1986) bekannt gewordenen Managementkreislauf repräsentiert das „Do“ die Ausführungsfunktion, das ,Study“ die Kontrollfunktion und das „Act" die von Erkenntnis geleitete Handlungsfunktion (siehe Abb. 2). Die in diesem Denk- und Handlungsmodell beschriebenen Funktionen und Aufgaben folgen einer ,hierarchisch-sequentiellen Ordnung" (Oesterreich 1981), d.h. die Aufgaben und Tätigkeiten sind sowohl aneinandergereiht und aufeinander aufbauend (sequentiell) als auch in übergeordnete Planungen eingebettet, die wiederum noch höher geordneten Planungen unterstehen (hierarchisch). Kontrolle als Managementfunktion ist hiernach abstrahierbar und modellierbar, von der Ebene der individuellen Handlungsregulation bis zur Steuerungsebene der Gesamtorganisation, als (Teil-)Funktion eines Handlungsmodells oder als (Gesamt-)Funktion zur Unternehmensführung.

Ein Kontrollbegriff, der in dieser Weise auf die Beherrschung von Gesamtsystemen (z.B. Unternehmen, Organisationen, Einrichtungen) wie von Teilsystemen (z.B. Prozesse, Projekte, Leistungseinheiten) gerichtet ist, ent- spricht einem systemtheoretisch-kybernetischen Verständnis (Regelkreissystem- und Rückkopplungs-Logik). Kontrolle wird darin als Managementfunktion konzipiert, die durch Stabilisierung und Optimierung primär der Sicherstellung der Planerreichung dient, abhängig vom Abstraktions- und Modellierungsgrad sekundär aber auch zur Korrektur oder Neuformulierung von Soll-Vorstellungen führen kann (siehe Abb. 1).

\subsection{Kontrolle als soziale Funktion}

Ein Systemdenken, das weniger die Systembeherrschung als vielmehr die Austauschbeziehungen der Systemkomponenten untereinander betont, liefert der systemtheoretischkonstruktivistische Denkansatz. Die „Plandeterminiertheit“ (Merchel 2012, S. 32) des Steuerungsdenkens mit dem Anspruch, das Unternehmensgeschehen kausal beeinflussen zu können, tritt zurück zugunsten einer Vorstellung, dass Organisationen ein „Eigenleben“ (Tacke und Drepper 2018, S. 28) besitzen, welches in hohem Maße von den Interessen und Interaktionen ihrer Mitglieder gestaltet wird (vgl. Wimmer 2011). Organisation selbst wird darin zu einer Form der 
„sozialen Systembildung“ (Lambers 2015, S. 32); Management als „reflexive Gestaltungspraxis“ (Rüegg-Stürm und Grand 2017, S. 33) konzipiert.

In dieser Konzipierung sind Menschen keine Produktionsfaktoren, die mechanistisch geführt werden müssen oder Gegenstand einer kybernetischen Steuerungslogik sind, sondern selbständig denkende und verantwortlich handelnde Individuen, die Planungs- und Kontrollansprüche für sich und ihr Arbeitsumfeld erheben. Im Zuge der „Humanisierung der Arbeit“ betrat Mitte bis Ende des 20. Jahrhunderts eine Vorstellung von Kontrolle die Bühne der Arbeits- und Organisationswissenschaften, die menschliche Bedürfnisse und Fähigkeiten stärker berücksichtigte. Mit Selbstkontrolle wurde eine Gegenposition zum tayloristischen Paradigma der Fremdkontrolle entworfen, die Verantwortung, Kompetenz und Autonomie als subjektbezogene Kategorien in die Arbeitsorganisation integrierte (vgl. Kieser 2014). Menschen sollten fortan mehr Selbstbestimmung und Möglichkeiten der persönlichen Entfaltung am Arbeitsplatz erhalten. Das Konzept der Selbstkontrolle beschränkt sich allerdings auf einen Modus der „kontrollierten Autonomie" (Vieth 1995). Dies bedeutet, dass Menschen, einzeln oder in Gruppen, mehr Selbstständigkeit und Verantwortung durch Hinzufügen von Entscheidungsund Kontrollaufgaben bei der Erledigung ihrer Realisationsaufgaben erhalten. Fremdkontrolle löst sich im Konzept der Selbstkontrolle nicht gänzlich auf, da zentrale Planungsvorgaben bzw. übergeordnete Zweckbestimmungen und Gestaltungsansprüche erhalten bleiben.

Hiervon abzugrenzen sind das Konzept von Selbstorganisation und das Phänomen Sozialer Kontrolle. Selbstkontrolle ist das Ausüben von Kontrolltätigkeiten durch Individuen oder Organisationseinheiten im Sinne einer zuvor beschriebenen Stabilisierungs- und Optimierungslogik zur Sicherstellung einer (übergeordneten) Planerreichung. Selbstorganisation geht über Selbstkontrolle hinaus, insofern sie planerische Aspekte miteinbezieht, die den Unternehmenszweck betreffen und die Geschäftstätigkeit beeinflussen. Dies bedeutet, dass Soll-Vorstellungen, beispielsweise legitimiert durch einen anerkannten Expertenstatus, auch zwecksetzend gebildet oder neuformuliert und sodann im Rahmen eines verfügten oder angeeigneten Handlungsspielraums (Kontrollbereich) gewissermaßen in ,Selbstverwaltung" realisiert und kontrolliert werden. Dies entspräche einem Modus von ,autonomer Kontrolle“, in dem Kontrolle als machtgebundenes Organisationshandeln abhängig von der Art und dem Umfang des handlungsleitenden „Sollens“ mit Gestaltungsmacht ausgestattet wird. Soziale Kontrolle wiederum beschreibt ebenfalls ein Gegenmodell zu Hierarchie und äußerem Zwang (Groenemeyer und Rosenbauer 2010). Kontrolle wird hierbei angeleitet und ausgeführt durch Tätigkeiten und Technologien „Gleichgesinnter“ und „Gleichgestellter“, die sich einen gemein- samen Werte-, Ziel- und Normenkanon teilen und miteinander Aufgaben erledigen (z. B. Peer-Teams, Expertenkreise, Teilnehmende in Arbeitsgruppen, Projektmitarbeitende). Stark vereinfachend zielt Soziale Kontrolle auf die Verhinderung von abweichendem Verhalten in einem sozialen System und konstituiert sich durch Reaktionen auf gegenwärtiges oder erwartetes abweichendes, d.h. normverletzendes Verhalten (Menzel und Wehrheim 2010, S. 511; ausführlich dazu Peters 2012). Sie rekurriert in hohem Maße auf die Selbstreflexion und Handlungsregulation des Individuums und wirkt über die formale Disziplinierung hinaus über die Subjektivierung bzw. Internalisierung von informellen Kontroll- und Sanktionsmustern. Dazu gehören beispielsweise Verhaltensmuster zur Vermeidung von Scham, Angst und Herabwürdigung oder zur Steigerung von Akzeptanz, Beliebtheit und Selbstwert. Durch Selbstkontrolle, Selbstorganisation und Soziale Kontrolle wird in verbindender Weise das Verhältnis von Fremd- und Selbstbestimmung zueinander konzeptualisiert. Trennend treten das Ausmaß an Autonomie und der Formalisierungsgrad der Kontrollsysteme in den Vordergrund.

Ein Kontrollbegriff, der nicht auf die Anwendung von Kontrollsystemen zur Beherrschung von Kontrollbereichen beschränkt bleibt bzw. bei dem das Organisationsgeschehen durch die Interaktion von verteilter Handlungs- und Gestaltungsmacht bestimmt wird, entspricht einem systemtheoretisch-konstruktivistischem Verständnis (Logik des sinn- und zweckgebenden Eigenlebens). Kontrolle erfüllt darin eine soziale Funktion des Interessenausgleichs und des Gestaltens von Gemeinschaft (siehe Abb. 1).

\section{Sach- und subjektbezogene Nutzenaspekte}

Mit der vorgenommenen Kategorisierung werden funktionale Aspekte aufgezeigt, die im Rahmen der Unternehmensführung oder Organisationsentwicklung unterschiedlich bedeutungsvoll hervortreten. Insbesondere die soziale Funktion von Kontrolle, die maßgeblich durch das Verhältnis von Fremd- und Selbstbestimmung geprägt wird, verlangt von den Organisationsmitgliedern eine Positionsbestimmung, welche „Rolle der Kontrolle“ den eigenen Handlungsmustern, Gestaltungsspielräumen und Entfaltungspotenzialen entspricht, bzw. ihr in den unterschiedlichen Anwendungszusammenhängen organisationalen Handelns zukommt. Es sind zuvorderst die handelnden Menschen, die Organisationen sinn- und zweckgebend „am Leben halten" und nicht die Instrumente, Steuerungsmechanismen und Ordnungsmuster. Eine solch hervorgehobene Rolle des Subjekts ist in einer funktionalen Betrachtung schon angelegt; sie begründet sich gewissermaßen von selbst. Für die eigene Positionsbestimmung ist es daher auch weniger ent- 


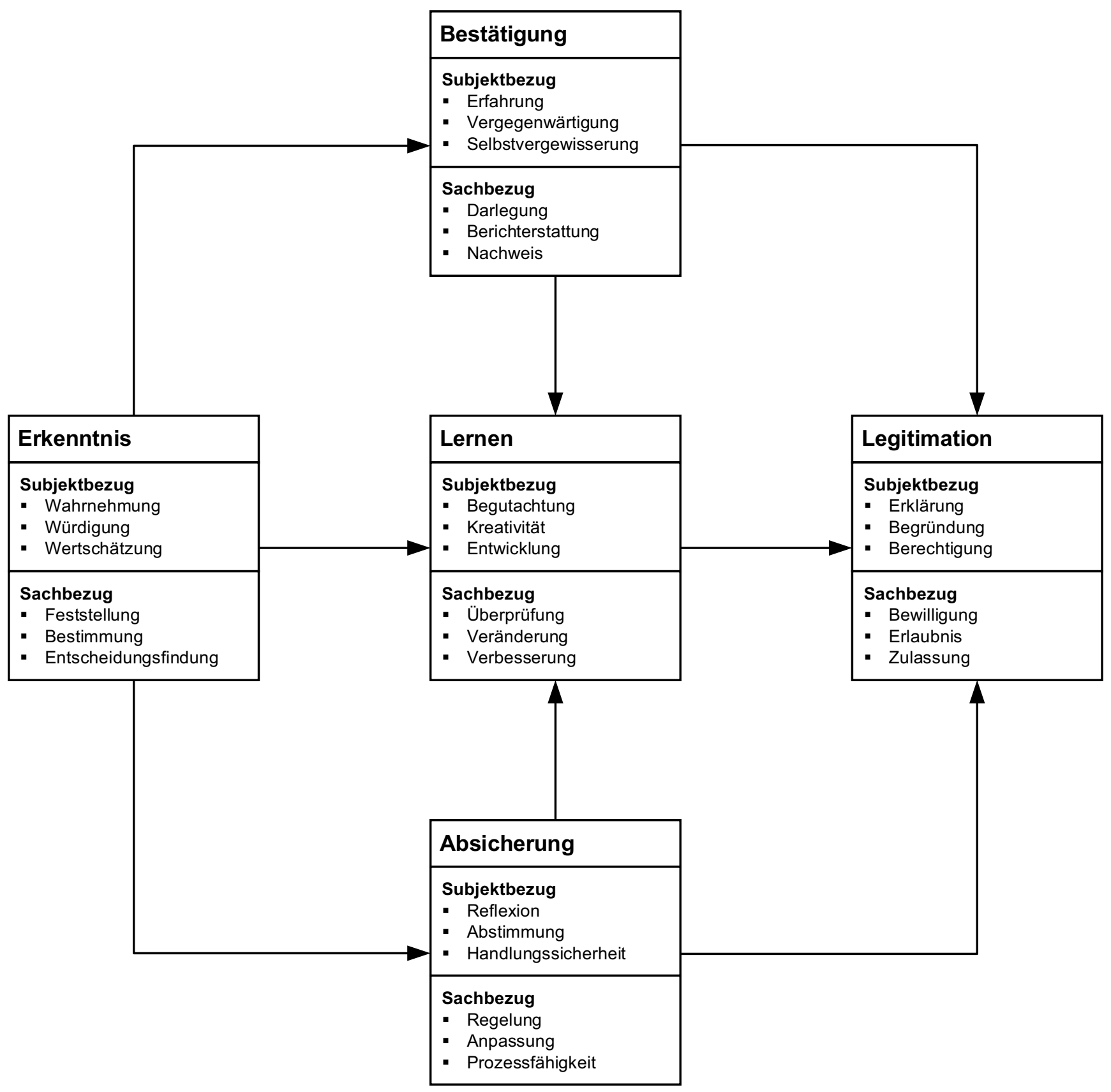

Abb. 3 Zusammenwirken sach- und subjektbezogener Nutzenaspekte von Kontrolle

scheidend, welche Aussagen „von außen“ hierzu getroffen werden, sondern wie die Organisationsmitglieder selber die „Rolle der Kontrolle“ in ihrem Arbeitsumfeld wahrnehmen und deuten.

Ein reflektiertes Verständnis von Kontrolle baut auf der Anerkennung ihrer sachbezogenen Bedeutung auf: bei der Erfüllung von Aufgaben, bei der Beherrschung eines Organisations- und Unternehmensgeschehens und als Beitrag von sinn- und zweckgebender Vergemeinschaftung. In der Einzelbetrachtung sachbezogener Nutzenaspekte zielt Kontrolle zunächst auf Erkenntnis. Sie umfasst einfache Fest- stellungen hinsichtlich der Anforderungserfüllung und einordnende Beurteilungen zur Funktions- und Wirkungsweise von Prozessen und Systemen. Gleichzeitig ist Kontrolle auf Veränderung gerichtet. Sie beinhaltet mehr oder weniger komplexe Ausführungen zur Verwirklichung von Absichten, gezielte Aktivitäten zur Behebung und Vermeidung von Abweichungen sowie Maßnahmen zur Vorbeugung von Gefahren und zur Verbesserung von Bedingungen. In diesem Sinne kann Kontrolle auch als Lernhandlung aufgefasst werden, mit der Wissen für nachfolgende Handlungen generiert wird (vgl. Schäffer 2001). Flankierend dazu 
dient Erkenntnis der Bestätigung. Darlegung und Berichterstattung (Reporting) transformiert Erkenntnisse in objektive Nachweise. Diese wiederum bilden das Substrat für interne Lern- und Veränderungsprozesse, aber auch der Legitimation gegenüber internen und externen Interessengruppen. Erkenntnis dient gleichzeitig der Absicherung, die wiederum ihrerseits regulativ und reaktiv für den Erhalt der Prozessfähigkeit steht. Die Überprüfung der systemischen Lebensbedingungen und Erhaltungssysteme wiederum ist Grundlage von Veränderung (Lernen) und dient gleichzeitig der Legitimation gegenüber Dritten (siehe Abb. 3).

Das Streben nach Erhaltung und Ausweitung von Kontrolle gehört zu den Grundbedürfnissen menschlichen Lebens (Fritsche et al. 2016). Im einem Verständnis von Organisation als ein soziotechnisches Handlungssystem (Ulich 2013), in dem Menschen selbstbestimmt und gestalterisch wirken wollen, ist Kontrolle nicht nur sachbezogen zur Durchsetzung betrieblicher Interessen begründbar. Sie kann für das handelnde Subjekt auch die im Folgenden beschriebenen und miteinander verbundenen Nutzenaspekte erfüllen (siehe Abb. 3):

- Erkenntnis: Absichtsvolles Handeln ist auf die Gewinnung von Informationen zur Erkenntnisbildung angewiesen. Ohne Feststellung, ob Ziele und Zwischenziele in der beabsichtigten Weise erreicht wurden, bliebe jede Handlung unvollständig. Kontrolle bedeutet hiernach, objektivierbare Aussagen über handlungsrelevante Sachverhalte zu erlangen. Die Arbeit von Individuen wird wahrnehmbar, sie kann gewürdigt und wertgeschätzt werden (,Erkenntnisbildung als Voraussetzung von Anerkennung").

- Bestätigung: Erkenntnis ist kein Selbstzweck. Die Erfassung und Beurteilung von Sachverhalten bildet die Grundlage individueller Erinnerung, welche sich als kompetenzorientierte Erfahrung verfestigt (,Verarbeiten von Erkenntnissen“). Diese wiederum wird situativ durch Vergegenwärtigung handlungsrelevant wirksam (,Erfahrungen für die Problemlösung nutzbar machen“) und erlangt als Selbstvergewisserung handlungsübergreifend Stabilität („Bestätigung als Handelnder und Rolleninhaber").

- Absicherung: Kontrolle entspricht dem Modus handlungsbegleitender Reflexion (,,Beurteilung und Regulation der eigenen Handlungen"). Dabei werden die eigenen Handlungsvollzüge und die Zusammenarbeit mit anderen Personen im Arbeitsumfeld sinnvoll aufund miteinander abgestimmt (,Erstellung anforderungsgerechter Leistungen"), wodurch Handlungssicherheit erzeugt wird (,In kritischen Situationen das Richtige tun und die richtigen Entscheidungen treffen").

- Lernen: Erkenntnisse, Erfahrungen und geübte Praxis stabilisieren Handlungen und Handlungssysteme. Kon- trolle ermöglicht darüber hinaus nicht nur, vorgängige Absichten $\mathrm{zu}$ verfolgen und festgelegte Anforderungen einer ,geschlossenen Zukunft“ zu erfüllen. Sie bedeutet auch, Handlungen hinsichtlich verändernder Wirklichkeiten zu begutachten (,,Sind die Mittel und Vorgehensweisen geeignet, um neuen Anforderungen zu entsprechen?") sowie Kreativität (,Potenziale schöpferischen Denkens und Handelns") für die Entwicklung der eigenen Handlungspraxis zu nutzen (,Veränderung und Erneuerung, die auch Nicht-Vorhergesehenes hervorbringt").

- Legitimation: Kontrolle ermöglicht Aussagen, Feststellungen und Nachweise darüber, was innerhalb der Organisation getan wird, wie der Umgang mit vorhandenen und verfügbaren Mitteln erfolgt und welcher Wirkungsgrad dabei erzielt wird. Es können sinnvolle Erklärungen für die Existenz, die Zuständigkeit und die Handlungsweisen von Menschen abgegeben werden. Sie liefert Begründung (,Wirkungsweise und Wirkungen von Handlungen“) und signalisiert Berechtigung (,Richtigkeit und Rechtmäßigkeit des Handelns") der eigenen Arbeit gegenüber Dritten.

\section{Kritische Einordnung}

Die formalen Eigenschaften des Kontrollbegriffs legen zunächst den Gedanken einer einheitlichen Begriffsanwendung nahe, doch treffen gerade innerhalb von Organisationen gegensätzliche oder sich wechselseitig beeinflussende Positionen aufeinander, die einer einebnenden Verwendung des Kontrollbegriffs bzw. einer vereinheitlichen Konzipierung entgegenstehen. Kontrolle als organisationaler Handlungsbegriff steht beispielsweise immer zwischen Machtausübung durch Führende (als Vertreter betrieblicher Anforderungen) und dem Kontrollempfinden der Mitarbeitenden (als Agenten der Anforderungserfüllung). In diesem Sinne wird Kontrolle zu einem graduellen Konzept, das sich durch seinen feststellbaren, mitunter ausschließlich subjektiv wahrnehmbaren Grad an Fremd- und Selbstbestimmung materialisiert. Determiniert wird Kontrolle weiterhin durch die Möglichkeiten der Kontrollausübung, die nicht nur vom Grad der zugesprochenen oder angeeigneten Macht „des Kontrollierenden“, sondern auch durch Art und Umfang der Gegenständlichkeit (d.h. Messbarkeit, Beschreibbarkeit, Abgrenzbarkeit) und Beeinflussbarkeit (d.h. Veränderbarkeit, Veränderungsbereitschaft, Veränderungsfähigkeit) „des Kontrollierten“ selbst limitiert werden. Die stabilisierende und optimierende Wirkung eines mechanistisch, kybernetisch oder konstruktivistisch geprägten Kontrollbegriffs wird stets durch das Verhältnis dieser Positionen zueinander bestimmt. Der Versuch, ein solches Verhältnis für einen bestimmten Anwendungszusammenhang 
konkret aufzuklären, bleibt auf den Beobachtungswinkel beschränkt und steht unter dem Vorbehalt von struktureller Situiertheit und interaktioneller Situativität des Handlungssystems. Für den Betrachter konstituiert sich Kontrolle aus jeder Position heraus neu und setzt sich nahezu unendlich ins Kleinste und Entlegenste fort, so wie sich beispielsweise Unternehmensziele bis auf die Wirkungsrichtung einer einzelnen Handbewegung theoretisch ,,herunterkontrollieren" ließen oder so wie Subjekt und Objekt der Kontrollausübung, ähnlich der in der Prozesstheorie geläufigen „Kunden-Lieferanten-Beziehung“, austauschbar sind, abhängig davon, welche Blickrichtung gewählt wird. Trotz des hier vorgenommenen Versuchs einer formgebenden und funktionalen Beschreibung lässt der Kontrollbegriff nahe$\mathrm{zu}$ beliebig viele Anwendungsoptionen zu und entzieht sich damit einem einheitlichen Verständnis und Umgang damit. Der Facettenreichtum von Kontrolle liegt weniger in der Unschärfe des Begriffs selbst begründet als vielmehr in der Uneinheitlichkeit und Mehrdeutigkeit seiner Anwendungsbezüge.

Als gemeinsam und beständig hinsichtlich der herausgelösten Nutzenaspekte kann dagegen die zentrale Rolle von Kontrolle als Lernhandlung betrachtet werden. Ein solches Verständnis ist anschlussfähig an den Bezugsrahmen lernender Organisationen nach Argyris und Schön (2002). Darin werden zwei Lerntypen unterschieden, die organisationales Lernen als Veränderungen erster und zweiter Ordnung konzipieren (vgl. Göhlich 2018). Vereinfachend zielt darin das Single-Loop-Learning auf Veränderung durch Regeln beispielsweise in Form von Fehlerkorrekturen, das Double-Loop-Learning auf Veränderung der Regeln, in dem Bedingungen, Strukturen und Mittel hinterfragt und verändert werden. Eine dritte, von Bateson (1981) als Deutero-Learning bezeichnete Lernebene, ergänzt die beiden anderen durch Reflexions- und Transformationsprozesse. In dieser dritten Lesart wird Lernen nicht nur als evolutiver Entwicklungsprozess verstanden, sondern auch im Sinne der Ausbildung und Weiterentwicklung einer organisationstypischen Lernkultur, gewissermaßen als Modus „lernenden Lernens“ (Wunderer 2000, S. 412) konzipiert. Geißler (2000) übersetzt diese drei Lerntypen in die Begrifflichkeit des operativen Anpassungslernens, des strategischen Erschließungslernens und des normativen Identitätslernens. Ausgehend von der Annahme, dass „Lernen“ als zentraler Nutzenaspekt den hier vorgenommenen Kontrollkonzeptionen zugrunde liegt, ließen sich diese in vergleichbarer Weise in die Grammatik des organisationalen Lernens übersetzen: Das mechanistische Verständnis von Kontrolle korrespondiert mit einem einschleifigen Anpassungslernen (,Prüf-und Korrekturlogik“), das kybernetische mit dem doppelschleifigen Erschließungslernen („Überprüfungs-und-Steuerungslogik“). Das Deutero-Learning wiederum käme der konstruktivistischen
Vorstellung einer sinn- und zweckvermittelnden sozialen Systembildung nahe. Eine solche Übersetzung weist den funktionalen Zugängen organisationale Handlungsebenen in Form von operativer Produktkontrolle (Ausführungsebene), strategischer Prozesskontrolle (Koordinationsebene) und normativer Systemkontrolle (Gestaltungsebene) zu.

Eine andere Deutung sähe in der vorgenommenen funktionalen Konzipierung keine Lern- und Reflexionsebenen, sondern vor allem Optiken, die Entwicklungsstufen offen legen und einen Bedeutungswandel von einem vertikalhierarchischen $\mathrm{zu}$ einem horizontal-partizipativen Kontrollverständnis markieren. Die als technisch bezeichnete Funktion des Kontrollbegriffs löst sich in den beiden anderen Betrachtungslinien jedoch nicht auf. Tätigkeiten wie „Produktprüfung“, „Prozessüberwachung“ oder „Anweisung zum Aufgabenvollzug" sind vielmehr eingebettet in höherwertige Vorstellungen von kybernetischer Systembeherrschung und sozialer Systembildung. Ihre ,herrschaftliche“ Bedeutung wird zugunsten von Verantwortung und Kompetenz in selbstkontrollierten bzw. selbstorganisierten Anwendungszusammenhängen gewissermaßen ,demokratisiert“. Vergleichbar dazu liefern kybernetische Ordnungsmuster und Regelkreise eine methodisch universelle Hintergrundfolie für die individuelle Handlungsregulation und für die kollektive Problembearbeitung. Sie sind nicht nur Handwerkszeug zur Durchsetzung einer zentralistischen Vorhabenplanung, sondern gleichzeitig auch Blaupause zur Verwirklichung eines interaktionell geprägten Organisationsgeschehens. Die hier vorgenommene Konzipierung soll daher auch keineswegs den Eindruck nahelegen, dass damit streng unterscheidbare oder sich gegenseitig ausschließende Kategorien vorgestellt werden.

Ein Zusammenspiel dieser drei Betrachtungslinien ist in besonderer Weise zu beobachten in Expertenorganisationen bzw. professionsgeleiteten Handlungssystemen, in denen wissens- und kompetenzintensive Dienstleistungen erstellt werden (z. B. Krankenhäuser, Unternehmensberatung, Bildungseinrichtungen). Hierarchische Kontrolle bildet sich in Parallelhierarchien innerhalb von unabhängigen, autonom agierenden Leistungssystemen ab. Die Leitungshierarchien werden ergänzt durch horizontale Verknüpfungen, durch die Wissen und Kompetenz ausgetauscht und für die Wertschöpfung nutzbar gemacht machen (Fach- und Kollegialitätsprinzip). Hierarchische Kontrolle wird hier sinnvoll verbunden mit autonomer und sozialer Kontrolle. Gleichzeitig durchdringen Regelkreis- und Rückkopplungssysteme die Gesamtorganisation. Sie bilden sich als professionelle Handlungsmuster auf Interaktionsebene, als Ordnungsmuster der Projekt- und Aufgabenrealisierung und als Managementsysteme auf Einrichtungs- und Unternehmensführungsebene ab. Die Expertenorganisation integriert verschiedene Kontrollkonzeptionen und veranschaulicht gleichzeitig den Übergang von einem eher 
herrschaftlich geprägten zu einem demokratisch geprägten Kontrollverständnis.

Dort, wo hierarchische und formelle Kontrollideen zugunsten von Verantwortungsübernahme und berufliche Handlungskompetenz zurücktreten, bestimmt das Beziehungskonstrukt Vertrauen das Verhältnis von Machtausübung und Kontrollempfinden. Redensartlich wird Vertrauen oft eine der Kontrolle nachgeordnete Rolle zugeschrieben. Dabei stehen Kontrolle und Vertrauen nicht kompetitiv, sondern in besonderer Weise komplementär zueinander (vgl. Eberl 2012). Dort wo Vertrauen herrscht, erscheint Kontrolle zunächst als unnötig. Ein augenscheinlicher Verzicht auf Kontrolle „,von außen“ bedeutet allerdings nicht gleichzeitig, dass sie „im Innern“ entbehrlich wäre. Vielmehr setzt Vertrauen Kontrolle als notwendige Bedingung geradezu voraus (Betzler und Scherrer 2017): ein Vertrauen, das darauf baut, dass etwas ,unter Kontrolle“ ist, dass Aufgaben erfüllt und Organisationen auf die eine oder andere Art funktionieren oder dass Werte, Normen und Regeln Geltung und Gültigkeit haben. Dieser Zusammenhang von „Vertrauen durch Kontrolle“ spiegelt sich auch in den zuvor aufgezeigten Nutzenaspekten. Kontrolle erzeugt dort Vertrauen, wo beispielsweise objektive Nachweise über die Leistungsfähigkeit und Handlungskompetenz bereitgestellt werden (Bestätigungsaspekt) oder wirkungsvolles Handeln gegenüber Dritten begründet und rechtfertigt wird (Legitimationsaspekt). Andererseits baut eine funktionale Konzipierung von Kontrolle auch ein Stück weit auf Vertrauen auf, beispielsweise durch ein Vertrauen auf den verantwortungsvollen Umgang mit verfügbarer Macht oder ein Vertrauen in die Selbstorganisationsfähigkeit und Verantwortungsbereitschaft jedes Einzeln (,Kontrolle durch Vertrauen").

Kontrollstreben gilt als menschliches Grundbedürfnis. Die psychosozialen Folgen von problembehaftetem Kontrollerleben bzw. von wahrnehmbarem Kontrollverlust sind vielfältig. Sie können zu unerwünschten Einstellungsund Verhaltensmustern wie Reaktanz und Hilflosigkeit führen oder sich nachteilig auf die physische und psychische Gesundheit bei den Betroffenen auswirken (vgl. Frey und Jonas 2002; Felfe 2012). Gleichzeitig ist nicht jeder Mensch bereit zu mehr Autonomie und Selbstbestimmung, die mit mehr Verantwortung einhergeht (vgl. ausführlich hierzu Frey und Jonas 2002; Fritsche et al. 2011). Für die Führung bedeutet das, Überforderung von Menschen durch unfreiwillige Verantwortungsdelegation genauso wie Rollenkonflikte durch mehrdeutige oder inadäquate Rollenanforderungen im Arbeitsumfeld zu vermeiden. Dazu gehört, das Ausmaß an vorhandener Verantwortungsbereitschaft und bestehendem Kontrollbedürfnis auf Seiten der Mitarbeitenden zu kennen und einen bestmöglichen Fit zwischen einem sachbezogenen „Sollen“ (betriebliche Erfüllungsansprüche) und „Dürfen“ (kontrollierte Autono- mie) sowie einem subjektbezogenem „Wollen“ (individuelle Kontrollansprüche) und „Können“ (Rollenkompetenz und Selbstorganisationsfähigkeit) herzustellen. Ein solcher Umgang mit Kontrolle heißt im Grunde nichts anderes, als widersprüchliche Interessen und Anforderungen dauerhaft mit den Instrumenten der Machtverteilung derart in Einklang zu bringen, dass zwar nicht jedes einzelne Kontrollbedürfnis beliebig und maximal erfüllt werden kann, Kontrolle jedoch möglichst wenig problembehaftet im Sinne von Kontrollverlust oder überfordernd im Sinne von Kontrollbelastung zu einem individuellen oder organisationalen Stressfaktor wird. Trauen wir insgesamt aber Menschen zu, verantwortungsvoll zu handeln, müssen wir sie darin unterstützen und förderliche Bedingungen schaffen. Das bedeutet, Kontrollfähigkeit bzw. den verantwortungsvollen Umgang mit verfügbarer Macht und den Umgang mit unterschiedlichen Kontrollbedürfnissen in die Personal- und Organisationsentwicklung bzw. betriebliche Kompetenzentwicklung zu integrieren. Dazu gehören insbesondere der Aufbau bzw. die Herstellung von:

- Kontrolltransparenz: Um Schwierigkeiten, Vorbehalten und möglichen Ängsten im Umgang mit erlebter und gelebter Kontrolle zu begegnen und kontraproduktives Arbeitsverhalten durch negative Zuschreibungen von Kontrolle zu vermeiden, sollte die Bedeutung des Kontrollbegriffs bzw. die damit verbundenen sach- und subjektbezogenen Nutzenaspekte jedem Organisationsmitglied zugänglich und verstehbar werden.

- Ambiguitätstoleranz: Die Gleichzeitigkeit und Vielschichtigkeit erlebter und gelebter Kontrolle geht zwangsläufig einher mit Widersprüchlichkeiten und Mehrdeutigkeiten, die zu Unsicherheiten bei der Aufgabenwahrnehmung und Verantwortungsübernahme führen können. Verantwortungsvolles Handeln setzt Fähigkeiten voraus, Gegensätze dieser Art in Organisationen wahrzunehmen, auszuhalten und auf mögliche Vereinbarkeit bspw. hinsichtlich des Verfolgens übergeordneter Ziele und Wertvorstellungen zu prüfen.

- Rollenklarheit: Eindeutigkeit bei der Zuordnung von Verantwortlichkeiten und Zuständigkeiten genauso wie die Klärung von Rollenanforderungen und die Verdeutlichung von Regeln erzeugt Verlässlichkeit und Orientierung. Selbstbestimmung und Verantwortungsübernahme bedeuten nicht, auf (kodifizierte) Regeln zu verzichten. Durch Rollenklarheit kann unerwünschten Phänomenen wie Verantwortungsdiffusion, Verselbstständigung und Vernachlässigung entgegen gewirkt werden.

\section{Fazit}

Ausgangspunkt der hier vorgenommenen Ausführungen war die Frage nach Anwendungs- und Bedeutungszusam- 
menhängen des Kontrollbegriffs und inwieweit sich Nutzenaspekte für die Organisationspraxis identifizieren lassen. Kontrolle wurde hierzu auf dreierlei Weise konzipiert: als technische Funktion zur Erfüllung von Sachaufgaben, als Managementfunktion zur Beherrschung von organisierten Handlungssystemen und als soziale Funktion zur Gestaltung von Gemeinschaft. Schlussfolgernd erscheint sie unentbehrlich für die Fähigkeit zur Leistungsbereitstellung, das Funktionieren von Prozessen, und die individuelle wie kollektive Problemlösung in komplexen Handlungsfeldern. In ihrer systemtheoretischen Betrachtung bleibt sie zunächst abstrakt; in ihrem Verwendungszusammenhang wird sie für jeden auf unterschiedliche Weise sichtbar und erfahrbar, insbesondere durch das wechselseitige Verhältnis von Kontrollausübung und Kontrollempfinden, von Fremdbestimmung und Selbstbestimmung bzw. von Macht und Möglichkeit. Ausgehend von formgebenden und funktionalen Beschreibungen wurden sach- und subjektbezogene Nutzenaspekte herausgelöst. Diese Nutzenaspekte können als Arbeitsfolie für die Organisationsanalyse und -beratung herangezogen werden, um das Verständnis für die Kontrollabsichten und -erfordernisse innerhalb von Organisationen und am eigenen Arbeitsumfeld zu erhöhen, Kontrollstreben sach- und subjektbezogen zu begründen und einem dysfunktionalen Kontrollempfinden von Zwang und Beeinträchtigung entgegenzuwirken.

Kontrolle ist stets geprägt von Ambivalenzen und Paradoxien, die auszuhalten sind. Dazu gehört die Gleichzeitigkeit von Systemerhalt und Entwicklungsoffenheit, das Nebeneinander von Stabilität und Innovationsfähigkeit aber auch die Konflikthaftigkeit objektivierbarer Unternehmenszwänge und subjektiver Gestaltungsansprüche. Kontrolle ist vieles, menschliches Grundbedürfnis und erfahrbare Macht, objektivierbarer Nutzen und subjektivierte Gefahr, als organisationaler Handlungsbegriff aber keineswegs entbehrlich. Wer sie ablehnt, trägt dazu bei, dass ohne sie falsches und nicht verantwortbares gedeiht.

Funding Open Access funding provided by Projekt DEAL.

Open Access Dieser Artikel wird unter der Creative Commons Namensnennung 4.0 International Lizenz veröffentlicht, welche die Nutzung, Vervielfältigung, Bearbeitung, Verbreitung und Wiedergabe in jeglichem Medium und Format erlaubt, sofern Sie den/die ursprünglichen Autor(en) und die Quelle ordnungsgemäß nennen, einen Link zur Creative Commons Lizenz beifügen und angeben, ob Änderungen vorgenommen wurden.

Die in diesem Artikel enthaltenen Bilder und sonstiges Drittmaterial unterliegen ebenfalls der genannten Creative Commons Lizenz, sofern sich aus der Abbildungslegende nichts anderes ergibt. Sofern das betreffende Material nicht unter der genannten Creative Commons Lizenz steht und die betreffende Handlung nicht nach gesetzlichen Vorschriften erlaubt ist, ist für die oben aufgeführten Weiterverwendungen des Materials die Einwilligung des jeweiligen Rechteinhabers einzuholen.

Weitere Details zur Lizenz entnehmen Sie bitte der Lizenzinformation auf http://creativecommons.org/licenses/by/4.0/deed.de.

\section{Literatur}

v. Ameln, F., \& Kramer, J. (2012). Macht und Führung. Gedanken zur Führung in einer komplexer werdenden Organisationslandschaft. Gruppendynamik und Organisationsberatung, 43, 189-204.

Argyris, C., \& Schön, D. (2002). Die lernende Organisation: Grundlage, Methode, Praxis. Stuttgart: Klett-Cotta.

Bateson, G. (1981). Ökologie des Geistes. Frankfurt a. M.: Suhrkamp.

Betzler, M., \& Scherrer, N. (2017). Verantwortung und Kontrolle. In L. Heidbrink, C. Langbehn \& J. Loh (Hrsg.), Handbuch Verantwortung (S. 337-352). Wiesbaden: Springer VS.

Bleicher, K. (Hrsg.). (1972). Organisation als System. Wiesbaden: Gabler.

Deming, W.E. (1994). The new economics for industry, government, education. Cambridge: MIT Press.

Eberl, P. (2012). Vertrauen und Kontrolle in Organisationen. In H. Möller (Hrsg.), Vertrauen in Organisationen. Riskante Vorleistung oder hoffnungsvolle Erwartung? (S. 93-110). Wiesbaden: VS.

Feigenbaum, A. V. (1962). Total quality control. New York: McGrawHill.

Felfe, J. (2012). Arbeits- und Organisationspsychologie 1: Arbeitsgestaltung, Motivation und Gesundheit. Stuttgart: Kohlhammer.

Fischer, J.H. (2004). Macht in Organisationen. Zu einigen Aspekten des Verhältnisses zwischen Individuum, Strukturen und Котmиnikationsprozessen. Münster: LIT.

Freidson, E. (1984). The changing nature of professional control. Annual Review of Sociology, 10, 1-20.

Frey, D., \& Jonas, E. (2002). Die Theorie der kognizierten Kontrolle. In D. Frey \& M. Irle (Hrsg.), Motivations-, Selbst- und Informationsverarbeitungstheorien. Theorien der Sozialpsychologie, (Bd. 3, S. 13-50). Bern: Huber.

Fritsche, I., Jonas, E., \& Frey, D. (2016). Das Bedürfnis nach Kontrolle als soziale Motivation. In H.-W. Bierhoff \& D. Frey (Hrsg.), Soziale Motive und soziale Einstellungen (S. 53-86). Göttingen: Hogrefe.

Fritsche, I., Jonas, E., Traut-Mattausch, E., \& Frey, D. (2011). Das Streben nach Kontrolle: Menschen zwischen Freiheit und Hilflosigkeit. In H.-W. Bierhoff \& D. Frey (Hrsg.), Sozialpsychologie - Individuum und soziale Welt (S. 85-110). Göttingen: Hogrefe.

Geißler, H. (2000). Organisationspädagogik. München: Vahlen.

Göhlich, M. (2018). Organisationales Lernen als zentraler Gegenstand der Organisationspädagogik. In M. Göhlich, A. Schröer \& S.M. Weber (Hrsg.), Handbuch Organisationspädagogik (S. 365-379). Wiesbaden: Springer VS.

Groenemeyer, A., \& Rosenbauer, N. (2010). Soziale personenbezogene Dienstleistungsorganisationen im Dispositiv der Kontrolle und Disziplinierung. In T. Klatetzki (Hrsg.), Soziale personenbezogene Dienstleistungsorganisationen. Soziologische Perspektiven (S. 61-102). Wiesbaden: VS.

Hochwarter, W. A., Rosen, C.C., Jordan, S.L., Ferris, G. R., Ejaz, A., $\&$ Maher, L.P. (2020). Perceptions of organizational politics research: past, present, and future. Journal of Management. https:// doi.org/10.1177/0149206319898506.

Horváth, P., Gleich, R., \& Seiter, M. (2015). Controlling (13. Aufl.). München: Vahlen.

Ishikawa, K. (1985). What is total quality control? New Jersey: Prentice Hall.

Ittel, A., Raufelder, D., \& Scheithauer, H. (2014). Soziale Lerntheorien. In L. Ahnert (Hrsg.), Theorien in der Entwicklungspsychologie. Berlin, Heidelberg: Springer VS.

Juran, J. M. (1995). A history of managing for quality - the evolution, trends and future directions of managing for quality. Milwaukee: American Society for Quality Control, Quality Press.

Jürgens, U. (1984). Die Entwicklung von Macht, Herrschaft und Kontrolle im Betrieb als politischer Prozeß: eine Problemskizze zur Arbeitspolitik. In U. Jürgens \& F. Naschold (Hrsg.), Arbeitspolitik: Materialien zum Zusammenhang von politischer Macht, Kon- 
trolle und betrieblicher Organisation der Arbeit (S. 58-91). Wiesbaden: Westdeutscher Verlag.

Kant, I. (o.J.). Kritik der Urteilskraft (KU), AA 05: 260.23.

Ketting, M. (1999). Geschichte des Qualitätsmanagements. In W. Masing (Hrsg.), Handbuch Qualitätsmanagement (4. Aufl. S. 17-30). München: Hanser.

Kieser, A. (2014). Managementlehren - von Regeln guter Praxis über den Taylorismus zur Human Relations Bewegung. In A. Kieser \& M. Ebers (Hrsg.), Organisationstheorien (7. Aufl. S. 73-117). Stuttgart: Kohlhammer.

Koontz, H., \& O'Donnell, C. (1955). Principles of management: an analysis of managerial functions. New York: McGraw-Hill.

Krist, T. (1997). Meß- Steuerungs- Regelungstechnik. Formeln, Daten und Begriffe (5. Aufl.). Braunschweig, Wiesbaden: Vieweg.

Lambers, H. (2015). Management in der Sozialen Arbeit und in der Sozialwirtschaft. Ein systemtheoretisch reflektiertes Managementmodell. Weinheim, Basel: Beltz Juventa.

Liesegang, D. G., \& Ullmann, K.M. (1994). Modellgestützte Regelkreise im Management. In B. Werners \& R. Gabriel (Hrsg.), Operations Research. Reflexionen aus Theorie und Praxis. Berlin, Heidelberg: Springer.

Matys, T. (2014). Macht, Kontrolle und Entscheidungen in Organisationen. Eine Einführung in organisationale Mikro-, Meso- und Makropolitik (2. Aufl.). Wiesbaden: Springer VS.

Meisler, G., Vigoda-Gadot, E., \& Drory, A. (2017). Stress, psychological strain, and reduced organizational effectiveness: the destructive consequences of the use of intimidation and pressure by supervisors. In C. C. Rosen \& P. L. Perrewé (Hrsg.), Power, politics, and political skill in job stress. Research in occupational stress and well being, (Bd. 15, S. 51-80). Bingley, UK: Emerald.

Menzel, B., \& Wehrheim, J. (2010). Soziologie Sozialer Kontrolle. In G. Kneer \& M. Schroer (Hrsg.), Handbuch Spezielle Soziologien (S. 509-524). Wiesbaden: VS.

Merchel, J. (2012). Der Managementbegriff im Gesundheits- und Sozialsektor. In G. Hensen \& P. Hensen (Hrsg.), Gesundheits- und Sozialmanagement. Leitbegriffe und Grundlagen modernen Managements (S. 21-37). Stuttgart: Kohlhammer.

Mirow, H. M. (1969). Kybernetik. Grundlage einer allgemeinen Theorie der Organisation. Wiesbaden: Gabler.

Oesterreich, R. (1981). Handlungsregulation und Kontrolle. München, Wien, Baltimore: Urban \& Schwarzenberg.

Peters, H. (2012). Soziale Kontrolle. In G. Albrecht \& A. Groenemeyer (Hrsg.), Handbuch soziale Probleme (2. Aufl. S. 1255-1284). Wiesbaden: VS.

Pries, L., Schmidt, R., \& Trinczek, R. (1990). Entwicklungspfade von Industriearbeit. Chancen und Risiken betrieblicher Produktionsmodernisierung. Opladen: Westdeutscher Verlag.

Richter, N. (2014). Organisation, Macht, Subjekt: Zur Genealogie des modernen Managements. Bielefeld: Transkript.
Rüegg-Stürm, J., \& Grand, S. (2017). Das St. Galler ManagementModell (3. Aufl.). Bern: Haupt.

Schäffer, U. (2001). Kontrolle als Lernprozess. Wiesbaden: Gabler.

Schildknecht, R. (1992). Total Quality Management - Konzeption und State of the Art. Frankfurt a.M.: Campus.

Schmitt, R., \& Pfeiffer, T. (2015). Qualitätsmanagement. Strategien Methoden - Techniken (5. Aufl.). München: Hanser.

Shewhart, W. A. (1986). Statistical method from the viewpoint of quality control. Washington D.C.: Dover Publications.

Spector, P.E., \& Fox, S. (2002). An emotion-centered model of voluntary work behavior: some parallels between counterproductive work behavior and organizational citizenship behavior. Human Resource Management Review, 12(2), 269-292.

Steinmann, H., Schreyögg, G., \& Koch, J. (2013). Management. Grundlagen der Unternehmensführung. Konzepte - FunktionenFallstudien (7. Aufl.). Wiesbaden: Springer Gabler.

Tacke, V., \& Drepper, T. (2018). Soziologie der Organisation. Wiesbaden: Springer VS.

Ulich, E. (2013). Arbeitssysteme als Soziotechnische Systeme - eine Erinnerung. Journal Psychologie des Alltagshandelns, 6(1), 4-12.

Ulrich, H. (1968). Die Unternehmung als produktives soziales System. Bern: Haupt.

Vieth, P. (1995). Kontrollierte Autonomie. Neue Herausforderungen für die Arbeitspsychologie. Heidelberg: Asanger.

Wild, J. (1982). Grundlagen der Unternehmungsplanung. Opladen: Westdeutscher Verlag.

Wimmer, R. (2011). Die Steuerung des Unsteuerbaren. In B. Pörksen (Hrsg.), Schlüsselwerke des Konstruktivismus (S. 520-547). Wiesbaden: VS

Wunderer, R. (2000). Führung und Zusammenarbeit: Eine unternehmerische Führungslehre (3. Aufl.). Neuwied: Luchterhand.

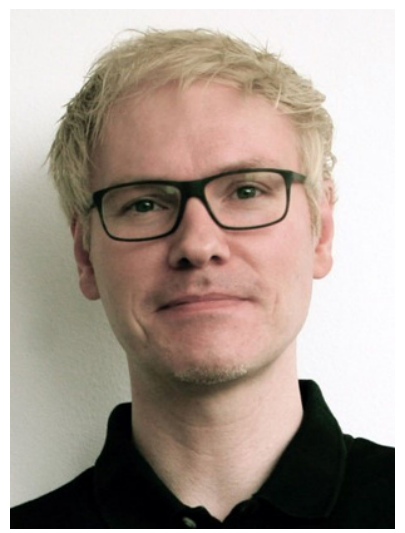

Prof. Dr. Peter Hensen ist Professor für Qualitätsentwicklung und -management im Gesundheits- und Sozialwesen an der Alice Salomon Hochschule Berlin. Schwerpunkte seiner Arbeit sind Theorie und Methoden des Qualitätsmanagements sowie Organisations- und Professionstheorie. 